\title{
Stretch effects of vortex tubes in vorticity fields for passive microdevice design.
}

\section{Arthur L. Valente ${ }^{\star}$, Sávio S. V. Vianna.}

\section{Abstract}

As far as microfluidics applications is concerned, either in the development of new products and processes or in the replacement of conventional procedures, the thorough comprehension of the physical phenomena which rule the flow of fluid inside micro-devices is of paramount importance. One of the main limitations is the time scale of mixture between fluids that is mainly governed by molecular diffusion. Computational fluid modelling arises as an interesting tool for both evaluation of the mixing index and optimisation of the micro-device geometry. The present work addresses the numerical modelling and investigation of flow in capillary scale for the development of micro-devices with emphasis in the process of formation of localised vorticity fields.

\section{Key words:}

Microfluidics, CFD, micromixer.

\section{Introduction}

Microfluidics, the flow study inside capillary dimension devices (10-100 micrometers), has grown immensely the past two decades because it shows potential in fields such as nanotechnology and biotechnology ${ }^{1}$. Due to their very small size, micro mixers work in laminar regime and rely crucially on diffusion, which it is not the ideal phenomenon for mixing two fluids. Therefore, for passive micro mixers, the geometry plays a huge factor creating chaotic advection ${ }^{2}$. In this scope, CFD gives an alternative, instead of experiments, to investigate the phenomena inside micro mixers, due to its effortless replicability and simplicity.

\section{Results and Discussion}

Image 1 points the details of the well known Staggered Herringbone and the novel inlet under development.

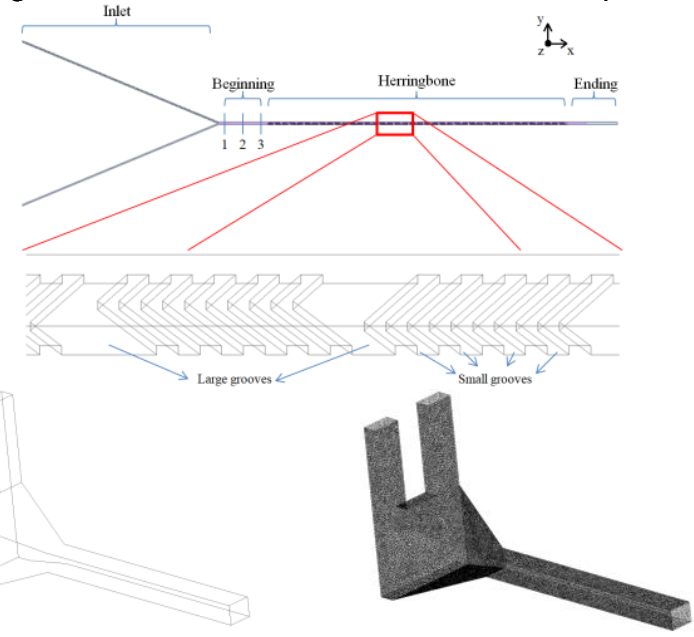

Image 1. Staggered Herringbone geometry pattern and novel geometry under investigation.

Results obtained from the novel device (Images 2 and 3 ) show positive expectancy in improving the mixing index.
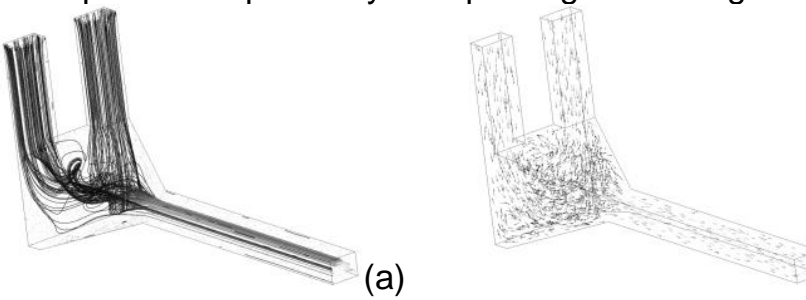

(a)

Image 2. Streamlines (a) and velocity vector field (b).
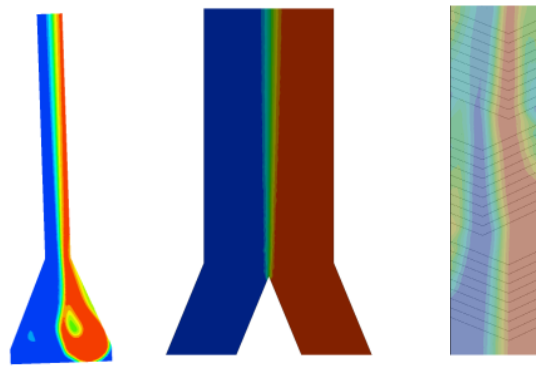

(a)
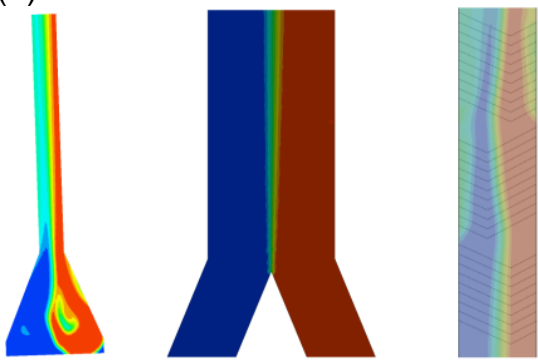

(b)
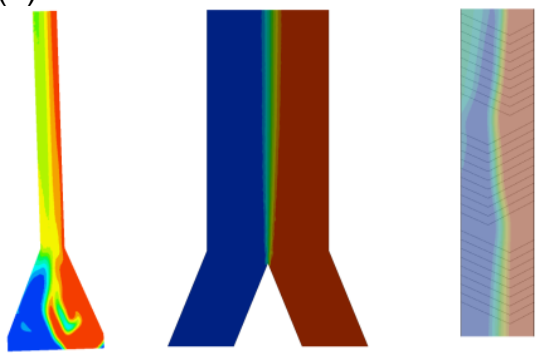

(c)

Image 3. Binary water-ethanol mixture at determined heights. (a): $30 \mu \mathrm{m}$. (b): $50 \mu \mathrm{m}$. (c): $70 \mu \mathrm{m}$.

\section{Conclusions}

Numerical findings show that the stretching of vortex tubes due to angular and momentum conservation enhance the mixture process leading to a novel micro mixer design based on Lapple ratios for cyclones.

\section{Acknowledgement}

Thanks are due to CNPq and also to FAPESP.

\footnotetext{
${ }^{1}$ Squires, T. M. Micro-plumes for nano-velocimetry.J. Fluid Mech., v. 832,p 1-4, 2017.

${ }^{2}$ Nguyen, Nam-trung; Wereley, Steven T..Fundamentals and Applications of
} Microfluidics. 2. ed. Norwood, Ma: Archtech House, Inc., 2006. 